\title{
IdeAs
}

Idées d'Amériques

$11 \mid 2018$

Modernités dans les Amériques : des avant-gardes à aujourd'hui

\section{Giulia Bogliolo Bruna. Les objets messagers de la pensée inuit}

Préface de Jean Malaurie, postface de Sylvie Dallet. Paris, L'Harmattan, Institut Charles Cros, 2015, 229 pages

Geneviève Chevallier

\section{QpenEdition}

\section{Journals}

Édition électronique

URL : https://journals.openedition.org/ideas/2301

DOI : 10.4000/ideas. 2301

ISSN : 1950-5701

Éditeur

Institut des Amériques

Référence électronique

Geneviève Chevallier, «Giulia Bogliolo Bruna. Les objets messagers de la pensée inuit », IdeAs [En ligne], 11 | 2018, mis en ligne le 14 novembre 2018, consulté le 19 octobre 2022. URL : http:// journals.openedition.org/ideas/2301; DOI : https://doi.org/10.4000/ideas.2301

Ce document a été généré automatiquement le 19 octobre 2022.

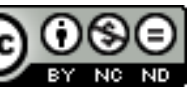

Creative Commons - Attribution - Pas d'Utilisation Commerciale - Pas de Modification 4.0 International - CC BY-NC-ND 4.0

https://creativecommons.org/licenses/by-nc-nd/4.0/ 


\section{Giulia Bogliolo Bruna. Les objets messagers de la pensée inuit}

Préface de Jean Malaurie, postface de Sylvie Dallet. Paris, L'Harmattan, Institut Charles Cros, 2015, 229 pages

\section{Geneviève Chevallier}

\section{RÉFÉRENCE}

Giulia Bogliolo Bruna. Les objets messagers de la pensée inuit. Préface de Jean Malaurie, postface de Sylvie Dallet. Paris, L'Harmattan, Institut Charles Cros, 2015, 229 pages

1 Suite à son précédent ouvrage intitulé Apparences trompeuses, Giulia Bogliolo Bruna aborde les objets inuit dans leur dimension polysémique en privilégiant leur essence symbolique. Ces objets révèlent une conception du monde, du temps et du rapport entre l'homme et le cosmos et sont appréhendés dans cette ontologie spécifique. L'analyse de l'auteure s'enroule autour de l'axe matière/esprit en décillant notre regard de prime abord esthétique.

2 Pour tous les découvreurs qui, au fil des siècles, s'aventurent dans l'Arctique, les Inuit demeurent inclassables dans la taxinomie pré-anthropologique de l'Occident et incarnent une Altérité qui bouscule toute épistémologie. Pour approcher le merveilleux nordique, l'auteur nous invite à percer les mystères de ces objets : miniatures, sculptures, talismans. "L'œil qui pense doit pénétrer dans leur système cognitif, adhérer à une intelligence cosmique régie par un vitalisme animiste... » (p.58).

3 La première partie, intitulée " quand la pensée chamanique se matérialise en objet", explore l'originalité de la pensée inuit issue d'un écosystème extrême et d'une vision chamanique du monde. Inter-mondes, l'œil du sacré, se réfère à la pensée mythique comme fondation d'un équilibre de vie. Nourris par leur fine observation de la nature, les peuples boréens imaginent un espace sacré en réponse à leur quête d'absolu dans lequel l'angakkoq, le chamane, régit les tabous et les rites associés pour le maintien de l'équilibre du monde. En considérant la sculpture - Sananguaq- ,comme le miroir de la 
pensée inuit, les miniatures apparaissent comme une transposition $\mathrm{du}$ monde surnaturel.

4 Le chapitre sur le Temps des origines, étudie la genèse de la pensée mythique du monde hyperboréen en remontant aux sources immémoriales. L'indifférenciation entre les règnes, les espèces et les sexes caractérise ce temps des Origines où l'androgynie apparaît comme l'état primordial. Le chapitre de fin, Dans le creux de la main, un monde en miniature aborde l'osmose entre les immensités de Nuna ( la Terre) avec les hommes du Grand Nord et les animaux qui la peuplent. Le rôle important des amulettes s'affirme dans cette nature instable, où les frontières entre le monde apparent et le monde invisible demeurent incertaines. La médiation de l'angakkoq est primordiale.

5 La deuxième partie de l'ouvrage est consacrée au « métissage » de la sculpture inuit. Intitulée Les temps mêlés des Rencontres : archéo-genèse et métamorphose des objets métis, elle rend compte des contacts progressifs entre Inuit et occidentaux, marquant le début du commerce et du métissage de l'art. L'expérimentation de l'altérité, la rencontre avec l'allaq, - l'autre - semble se décliner dans un pragmatisme naturel dynamisé par un échange de biens. "Les Inuit n'ont jamais été enfermés dans un destin de solitude... Depuis l'aube des temps, ils bénéficiaient d'un double réseau de commerce : continental du Groenland au Labrador, du Nord-Est jusqu'à l'Alaska et intercontinental de la Sibérie à l'Amérique du Nord-Ouest» (p. 124). Les artefacts attestent de ces rencontres interculturelles, notamment par l'apparition de la croix sur les figurines. Des Vikings aux baleiniers, jusqu'aux marchands du XIX ème siècle, les contacts se multiplient, s'intensifient et les objets inuit s'inscrivent comme mémoire matérielle de l'histoire du métissage en marche.

6 L'auteur constate que l'apport des Européens induit une transformation de la pratique sculpturale cependant «les emprunts et les prêts demeurent sélectifs, fidèles à une logique d'utilité, d'opportunité et de compatibilité »(P.127). L'influence des matériaux exogènes sur la praxis traditionnelle ainsi que l'intrusion d'exigences nouvelles pour satisfaire les lois d'un marché naissant, modifient irréversiblement la création des miniatures. Toutefois, la production sérielle ne dénature pas l'art inuit. Même dépossédées de leur pouvoir chamanique, les miniatures respectent le style naturaliste qui demeure le sceau de l'art inuit.

7 Dans le paragraphe intitulé Aux marges de l'oecoumène: Ultima Thulé, l'ethno-historienne évoque l'effroi et l'attraction suscités par l'apparition des grands voiliers aux yeux des Inuit. Véritables métaphores de l'étrange qui flottent sur les eaux arctiques, ces navires bouleversent l'imaginaire autochtone. Un Esquimau rencontré lors de l'expédition de John Ross vers 1819 s'interroge : « Qui êtes-vous ? D'où venez-vous ? de la Lune ou du Soleil ?»(p.148).

$8 \mathrm{Au}$ fil des escales, les échanges se multiplient, la communication s'installe, le mimétisme des hommes du Nord leur permet de s'adapter à une nouvelle temporalité et la dépendance s'inscrit graduellement. Des phénomènes de syncrétisme religieux apparaissent, remplaçant peu à peu le chant de l'angakkoq. L'objet messager retrace toute l'histoire de la Rencontre. Cependant, selon l'auteur, « au travers des processus d'escamotage ou par allusion métonymique, il dévoile les survivances/résurgences d'une pensée chamanique millénaire.» ( p. 156).

9 L'hybridation des matières et des techniques, issue du contact avec la culture et la technologie des Blancs, aboutit à la création de nouveaux objets répondant aux « logiques métisses ». L'objet devient médiateur culturel, attestant des transferts de 
savoir, avec l'imbrication de deux pensées, et la juxtaposition de deux histoires qui cherchent une forme d'alliance. Il est le produit d'une dynamique d'échange et d'une volonté de dialogue défiant la taxonomie car il relève d'une catégorie d'humanité nouvelle : celle du Contact.

Nous pouvons envisager la production matérielle née du métissage comme l'expression d'une volonté indigène de soumettre l'Allaq à une logique autochtone qui transforme les réalités allogènes en les «indigénéisant »: «Bien que nés de la Rencontre, ces objets métis continuent de répondre à une "esthétique de la fonctionnalité " par laquelle se marient les " utilités »: matérielles et symboliques.»(p.169). Toutefois, dans le domaine du sacré, le syncrétisme entre pratiques chamaniques ancestrales et évangélisation complexifie la nature et l'esthétique des objets.

11 Dans ce contexte, les Inuit du Groenland créent les tupilait, figurines issues de l'hybridité culturelle. Le tupilak représente un esprit malfaisant, condensé de forces occultes, destiné à nuire dans le cadre d'une vengeance. Cette créature est l'œuvre d'un ilisitsok (le double maléfique de l'angakkoq) qui la fabrique sur ordonnance dans une stricte confidentialité. La puissance esthétique du tupilak consiste dans l' hybridité homme-animal doublée d'androgynie associée à une savante disproportion des formes et à un expressionnisme exalté. L'exubérance triomphante de cette invention des kalaallit signe l'apothéose de la Rencontre et la fin de la période historique. Le tupilak devient l'image paradigmatique de l'art métis du Groenland, et se transforme en objet de curiosité fort prisé par les collectionneurs.

En conclusion, l'auteur rend hommage à l'ingéniosité des chasseurs-sculpteurs en insistant sur le dynamisme de la pensée inuit et suggère de lire, à travers l'évolution des objets inuit, l'expression «d'une culture de l'oralité insérée dans le flux temporel... » (p.209). Cette proposition nous engage à voir au-delà du visible, portés par l'enthousiasme que la connaissance de l'Autre suscite, en décolonisant notre regard pour accéder à un enchantement éclairé. Nous souhaiterions voir cet essai développer l'évolution de l'esthétique inuit jusqu'à la période contemporaine pour analyser les survivances chamaniques dans l'art des Inuit du Canada et des Yupiks d'Alaska.

\section{AUTEURS}

\section{GENEVIÈVE CHEVALLIER}

Centre d'Études Arctiques (CEARC). Université de Versailles Saint-Quentin-en-Yvelines /

jgchevallier@gmail.com 